\title{
Servicios Municipales de Atención Comunitaria Psicológica y Educativa: Una Experiencia de Atención Comunitaria Psicológica y Educativa y Perspectivas de Futuro
}

\section{Municipal Psychological and Educational Community Care Services: An Experience in Community Psychological and Educational Care and Prospects for the Future}

\author{
José A. Salgado Ballestero \\ Ayto. de San Sebastián de los Reyes, España
}

\author{
Fernando Sardinero Sac \\ Ayto. de Móstoles, España
}
Enrique Gallego Gallardo
Ayto. de Getafe, España

\begin{abstract}
Resumen. En este trabajo se recoge la experiencia de los Servicios de Atención Comunitaria Psicológica y Educativa que, desde un ámbito municipal, vienen desarrollando distintos programas de intervención y actividades de carácter preventivo y comunitario. Dichas actividades parten de un análisis de necesidades que permiten una actualización permanente de sus objetivos de actuación y basado en un modelo de competencia y metodologías evaluables. Este servicio cubren necesidades y un espacio de trabajo determinado para el que no hay respuesta desde ningún otro servicio u organismo.

Palabras clave: atención comunitaria, Psicología Educativa, servicios psicopedagógicos municipales.
\end{abstract}

\begin{abstract}
This paper describes the experience of Community Care Services and Educational Psychology, which, on a municipal level, are developing different intervention programs and preventive community activities. These activities are based on a needs analysis to enable the continuous updating of performance targets and are based on a competency model and evaluable methodology. This service covers an area of needs and a specific job for which there is currently no response from any other department or agency.

Keywords: community care services, Educational Psychology, municipal psychopedagogical services.
\end{abstract}

La realidad socioeducativa de la Comunidad de Madrid ha representado uno de los planteamientos más interesantes de los últimos años y uno de los retos de educación no formal para los psicólogos educativos. En este trabajo pretendemos plasmar la historia de la intervención psicoeducativa en los aspectos no estrictamente escolares, contemplada desde la perspectiva de los profesionales que trabajamos en el ámbito educativo con dependencia municipal. Describiremos los cambios que, en nues-

La correspondencia sobre el artículo debe enviarse al primer autor al e-mail:salgado@cop.es tra opinión, se ha producido en nuestra intervención y desempeño profesional, y que han venido determinados por el desarrollo de las diferentes leyes de Educación, los criterios políticos y la organización de las competencias administrativas en el periodo comprendido entre el establecimiento de la democracia hasta nuestros días.

A partir de 1979 y con la aparición de los Ayuntamientos democráticos se constituyen los Servicios Psicopedagógicos Municipales. Los primeros referentes se producen en Cataluña, donde se venía trabajando desde un modelo comunitario que ha caracterizado el trabajo de estos equipos y que 
provenía de experiencias desarrolladas en otros países europeos, como Francia, Italia o Gran Bretaña. Los Servicios fueron creándose en distintos lugares del territorio español, en algunos casos por necesidades comunes a todos los municipios y en otros por otras de carácter específico.

En el caso de la Comunidad de Madrid, en diferentes áreas metropolitanas y distritos de la capital se desarrollaron a partir de 1980. Los servicios eran de dependencia municipal y su finalidad era la de servir de instrumento técnico de apoyo en materia educativa a la comunidad local, planteándose como respuesta a las necesidades de la población, especialmente la escolar, con una deficitaria situación de la educación obligatoria, que se mostraba carente de recursos para afrontar los altos índices de fracaso escolar. En el caso concreto de Madrid, los cinturones industriales de la periferia eran el caldo de cultivo para el desarrollo de bolsas de marginalidad entre los jóvenes. Los programas se han desarrollado en la sede de los propios servicios, en los centros educativos de Educación Infantil, Primaria o Secundaria o en otros recursos y estructuras municipales.

Por las variaciones en la creación, desarrollo y mantenimiento de los equipos en éste largo periodo, resulta difícil de calcular la población a la que se dirigen los programas. Por recoger datos actualizados, los equipos atienden a una población potencial de más de 1.211.000 ciudadanos y específicamente a un rango de edad de menores de 0 a 16 años que se estima mayor de 205.000 menores. Al tener como población objetivo toda la comunidad educativa, habría que tener en cuenta también a las familias de estos menores, su profesorado y el resto de recursos con los que se establece coordinación.

\section{Objetivos y actividades de los Servicios Psicopedagógicos Municipales}

La actividad inicial y mayoritaria se concentró en el desarrollo de programas de intervención, apoyo y formación del profesorado, apoyo psicopedagógico, etc., ante la ausencia total de este tipo de servicios en la Administración Pública (Estatal y Autonómica). Esta actividad tenía como ejes de trabajo la defensa de la escuela pública, el apoyo a la renovación pedagógica de nuestras escuelas, el acercamiento del sistema educativo a la ciudad y la integración escolar, promoviendo la calidad de la educación, la participación del profesorado, de los padres y de los alumnos, el apoyo y atención al alumnado en situación de mayor desventaja social y con dificultades de adaptación a su entorno y necesidades educativas especiales. No obstante, otro de sus objetivos fue ofrecer a la población y especialmente a la escolar la atención psicológica necesaria, apoyando a los niños y niñas en el proceso de desarrollo integral de su personalidad, en los aspectos emocional, escolar y social, todo ello a través de los agentes educativos principales: la escuela y la familia. Estos servicios municipales han ido cambiando su denominación en función de las nuevas realidades locales y la aparición de servicios de otras administraciones.

La metodología de su intervención se ha caracterizado por un enfoque preventivo y comunitario, que incluye un análisis de necesidades como parte de la intervención, que engloba el análisis de la demanda y la formulación de objetivos, seguidos de procesos de valoración y evaluación, así como de comunicación de resultados a la comunidad educativa y responsables institucional.

Como en otros aspectos, en el educativo el ámbito local ha abierto brecha y ha desarrollado siempre estrategias de carácter innovador en la oferta de más y mejores servicios para la población que, posteriormente, han sido imitados $\mathrm{u}$ ofertados por otras instancias. Así, el trabajo de estos equipos se ha ido adaptando a nuevas demandas y necesidades, a la realidad de cada municipio, a diferencia de otros equipos creados por las administraciones estatal y autonómica, que intervienen en la comunidad educativa y que marcan determinadas áreas de intervención y prioridades con una metodología mucho más rígida y una mayor dificultad para reformular tareas y demandas. La multiplicidad de tareas y programas 
que se realizan desde los equipos viene recogida en las tablas 1,2, 3 y 4 . También pueden consultarse las páginas webs de los distintos ayuntamientos referidos.

Los programas y actividades desarrollados han permitido trabajar junto al profesorado en la motivación de sus alumnos, adaptando las necesidades educativas y diseñando estrategias, mejorando el clima del aula o aumentando el control de conductas desadaptativas, permitiendo con todo ello la mejora en los procesos de enseñanza y aprendizaje. Se ha promovido igualmente el desarrollo de aspectos de salud, desde acciones de promoción y prevención primaria, secundaria y terciaria, previendo dificultades o conflictos relacionados con la salud conductual, social y emocional de los niños, los grupos o los centros, con una especial intervención en la formación de familias, para mejorar el clima familiar, disminuir las dificultades, prevenir e intervenir en el absentismo escolar, etc. (véase tabla 1).

Se ha trabajado con la comunidad educativa en general, para promover ambientes educativos y de clima escolar que favorezca el aprendizaje y evite la aparición de conflictos, con estrategias específicas de fomento de la participación, para la reducción de la violencia, el bullying o el acoso. Se ha intervenido también en situaciones de emergencias, ante sucesos que podían afectar a la población infantil o a sus familias, así como en la coordinación con otros agentes, servicios o recursos comunitarios. Otro aspecto específico de los equipos ha sido el trabajo preventivo con las familias, mediante programas de Educación para la Salud, Prevención Primaria, Secundaria y Terciaria, con el desarrollo y puesta en práctica de formatos, modos de evaluación e intervención y materiales de apoyo específicos.

Por su integración en la Comunidad Educativa, los equipos han participado, dinamizado o liderado distintas investigaciones aplicadas que han permitido conocer e intervenir en la realidad de los menores en distintos municipios, colaborando con distintas Universidades o Escuelas de Formación e instituciones de muy diverso tipo (Red de Ciudades Educadoras, Red a favor de los Derechos de la
Infancia y la Adolescencia, UNICEF, Federación de Municipios y Provincias, Instituto Universitario de Necesidades y Derechos de la Infancia y la Adolescencia, Asociación Española de Normalización y Certificación, Instituto Tecnológico del Juguete, etc.) (véase tabla 2).

Los equipos han ido desarrollando distintas acciones de investigación, formativas y profesionales mediante la participación de distintos recursos (a través de convenios con Universidades, con los Centros de Orientación y Empleo, como espacios para la realización del Practicum y de prácticas, mediante el establecimiento de convenios como Corporaciones Locales para la contratación de trabajadores desempleados en la realización de obras y servicios de interés general y social, etc.). Los Servicios Municipales siguen realizando tareas de intervención psicoeducativa, junto a las de gestión, que fueron asumiéndose a lo largo del tiempo (véase tablas 3 y 4).

Estos equipos tienen como finalidad detectar las nuevas y más cercanas necesidades de los ciudadanos y, a la vez, ser capaces de diseñar programas que den respuesta a estas necesidades, lejos de la rigidez normativa de los equipos de otras administraciones no tan cercanas a los ciudadanos. Con los cambios sociales cambian las necesidades de los ciudadanos y la disponibilidad de recursos económicos y profesionales. Ello explica, al menos en parte, la vigencia de los Servicios Psicopedagógicos. Estos servicios ya han dado respuesta a nuevas situaciones, realidades y demandas tales como el trabajo con población en situaciones de riesgo, de interculturalidad, problemas emergentes como acoso, violencia entre iguales, nuevas realidades familiares, lo que ha facilitando el desarrollo de ciudades educadoras, más participativas y sostenibles, fomentando la sociedad del conocimiento, la comunicación y la incorporación de las nuevas tecnologías. Por ello, estos servicios van más allá de las necesidades de una época concreta. Son equipos estables y, al mismo tiempo, flexibles, adaptables a las necesidades concretas de la población (véase tabla 5). 
Tabla I. Programas desarrollados desde los servicios municipales de astención comunitaria psicológica y educativa

\section{PROGRAMAS DE ATENCIÓN PSICOLÓGICA Y EDUCATIVA}

Relacionados con información y asesoramiento, aprendizajes, formación de padres, orientación escolar y profesional, temas transversales, adquisición de habilidades, normalización, asistencia psicoeducativa, asesoramiento psicológico individual a jóvenes y familias

Relacionados con el apoyo al profesorado, a través de las propuestas de la psicología educativa en clave de competencias y habilidades y el trabajo con las familias, para proveer a los padres de criterios y recursos y actuar en el entorno familiar

\begin{tabular}{ll}
\hline Relacionados con información y asesoramiento & Información y asesoramiento psicológico y educativo \\
& Orientación psicoeducativa individual \\
& Orientación/ información de recursos \\
Intervención familiar
\end{tabular}

Relacionados con aprendizajes

Intervención familiar

\begin{tabular}{ll} 
Relacionados con aprendizajes & Apoyo educativo \\
Intervención psicopedagógica & Valoración, orientación y programación psicopedagógica de alumnos con problemas de \\
aprendizaje & Reeducación logopédica \\
Intervención en dificultades para el aprendizaje de la lectoescritura & Mejora de hábitos lectores \\
Comprensión lectora & Técnicas y hábitos de estudio \\
\hline Relacionados con la formación de padres & Escuelas de padres y madres \\
Mejora de las habilidades para educar a los hijos \\
Mejora de las relaciones familiares \\
Seminario de adolescencia \\
Intervención en problemas de conducta (enuresis, encopresis, dislalias, dificultades en \\
la relación social, hipercactividad, trastornos del sueño, miedos, problemas con las comi- \\
das, ...) \\
Estimulación del lenguaje \\
Hábitos de alimentación \\
Apoyo a las tareas escolares
\end{tabular}

Relacionados con la orientación escolar y profesional Orientación escolar (en los diferentes niveles educativos)

Orientación a alumnos de escuelas taller

Orientación académico-profesional

Orientación e inserción socioprofesional

Relacionados con temas transversales

Promoción del desarrollo de programas de educación para la salud en los centros educativos

Salud escolar

Educación afectivo-sexual

Educación para la igualdad de oportunidades en ambos sexos

Educación ambiental

Cultura y municipio

Educación vial

Taller de juego cooperativo

Relacionados con adquisición de habilidades

Intervención para la adquisición de hábitos de autonomía

Desarrollo a través del juego

Control de ansiedad ante exámenes

Desarrollo de la autoestima

Mejora de las habilidades sociales

Mejora de las relaciones sociales y comunicativas en adolescentes

Relacionados con la normalización

Relacionados con la escolarización

Relacionados con actividades y Planes de Mejora
Prevención de la conducta agresiva en el aula

Prevención y detección de absentismo escolar y otras situaciones de riesgo en la infancia Apoyo a niños en situación de riesgo psicosocial

Prevención e intervención del alumnado de E.S.O. de riesgo psicosocial

Apoyo a la escolarización de niños de otras nacionalidades o con necesidades de compensación educativa

Programas de Garantía Social

Inserción sociolaboral de alumnos de riesgo

Escolarización

Selección de alumnos para programas de Garantía Social

Apoyo extraescolar en temas educativos para Educación Primaria Apoyo extraescolar en temas educativos para Educación Secundaria Ludotecas

Talleres de juego 
Tabla 2. Programas de participación en la comunidad educativa

Relacionados con el fomento de la participación en la escuela, la infancia, las AMPAs, el alumnado y el profesorado, generando cauces de comunicación entre la comunidad y la escuela

\section{Relacionados con la escuela}

Participación en Consejos Escolares

Participación en Consejo Sectorial de Educación

Participación en Consejo Escolar Municipal

Participación en Comisiones de Seguimiento de Planes de Mejora

\section{Relacionados con la infancia}

Difusión e información de las actividades de los S.M.A.C.P.E.s

Jornadas sobre temas educativos

Participación en actividades y movimientos asociativos

Formación y asesoramiento a asociaciones de padres/ madres de alumnos

Formación y asesoramiento a Representantes Municipales en Consejos Escolares

Apoyo técnico al Consejo Municipal de Educación

Gestión de subvenciones

Subvención a proyectos educativos

Tabla 3. Programas de coordinación de recursos

Relacionados con la coordinación de recursos, la coordinación interinstitucional, con convenios, con participación en Redes (Red a favor de los derechos de la Infancia y la Adolescencia, Red de Ciudades Educadoras, Coordinadora de Servicios Municipales de Atención Comunitaria Psicológica y Educativa, Coordinadora de Ludotecas Municipales, Mesa de Absentismo, Mesa de Salud Escolar, etc.)

Relacionados con la coordinación de recursos

Coordinación con recursos municipales

Coordinación con recursos públicos

Coordinación con recursos privados

\section{Relacionados con la coordinación interinstitucional Programa de Educación para la Salud}

Prevención de drogodependencias

Prevención VIH/ SIDA

Comisiones y coordinadoras de atención a la infancia y la adolescencia

Colaboración con programas para la incorporación laboral de jóvenes

Relacionados con Convenios

Establecimiento de convenios con distintas instituciones para el desarrollo de programas Establecimiento de convenios para la incorporación de alumnos en prácticas

Tabla 4. Programas de investigación y desarrollo

Relacionados con Infancia y Adolescencia, con población general, con participación

Relacionados con infancia y adolescencia
Detección e incidencias de riesgo biopsicosocial en niños y adolescentes

Prevención e intervención en factores de riesgo en adolescentes

Estudio sobre las necesidades psicoeducativas y sociales de los adolescentes

Estudio sobre los conocimientos y mitos sexuales en chicos y chicas adolescentes

Trabajo preventivo y de salud en $1^{\circ}$ y $3^{\circ}$ de Educación Secundaria Obligatoria

Estudio sobre la implementación de la Educación Secundaria Obligatoria

Estudio sobre el daltonismo

Evolución del sexo y el género de 3 a 7 años

Estudio de la conducta antisocial en el periodo escolar

\section{Relacionados con la población general}

Análisis epidemiológico

Relacionados con la participación

Estudio sobre la participación educativa a través de los Consejos Escolares

Tabla 5. Otros programas

Planes integrales para la Infancia y Adolescencia, de conocimiento de la ciudad, de prevención e intervención en absentismo escolar, de apoyo a alumnado en riesgo, de salud para adolescentes, de entrenamiento con alumnado en familias (en habilidades personales, sociales, trastornos de alimentación), de prevención del acoso escolar y de convivencia, de conciliación y coeducación, en Planes de Mejora y Extensión de los Servicios Educativos (tareas de apoyo escolar en Infantil, Primaria y Secundaria, actividades de conciliación en horario extraescolar, en días no lectivos en periodos de vacaciones escolares), etc. 


\section{Composición de los equipos psicopedagógicos}

La composición de los equipos es variada, tanto en el número de sus componentes como en los profesionales que los integran, contando según los casos con psicólogos, pedagogos, trabajadores sociales, personal administrativo y subalterno. Esta disposición de profesionales ha estado sometida a múltiples variaciones a lo largo de estos años, disminuyendo el personal contratado por el propio ayuntamiento y aumentando las contrataciones externas, a través de oferta para cubrir servicios, convenios y subvenciones. Esta composición también afecta a las tareas desarrolladas así que, mientras en algunos servicios se ha mantenido la intervención psicoeducativa como eje principal de su actuación otros, en cambio, han ido evolucionando hacia el desarrollo de tareas de gestión. Aunque ello ha permitido una mayor extensión y cobertura de la población, también ha aumentado la complejidad, las dificultades de control, las tareas de coordinación y han hecho imprescindible la definición de criterios de calidad para poder seguir manteniendo, en lo posible, unos niveles adecuados de globalidad y estabilidad en las intervenciones, en cuanto que los equipos siguen manteniendo la historia y el análisis longitudinal de las necesidades y las intervenciones propuestas.

La Coordinadora de Servicios Municipales de Atención Comunitaria Psicológica y Educativa ha funcionado como un grupo estable de profesionales. En el momento actual, la Coordinadora está formada por técnicos de los equipos municipales de Alcorcón, Colmenar Viejo, Fuenlabrada, Getafe, Leganés, Móstoles, Parla, San Sebastián de los Reyes y Valdemoro y tienen, como objetivos fundamentales, los siguientes:

- Reivindicar un espacio profesional propio, caracterizado por una perspectiva comunitaria.

- Propiciar el intercambio sobre programas dirigidos a la infancia y la adolescencia y a la comunidad educativa en general.

- Facilitar espacios comunes de formación.

- Desarrollar líneas de investigación, desarrollo e innovación mediante investigaciones aplicadas.

\section{Perspectivas de futuro}

La comunidad educativa requiere también en el momento actual abordar los aspectos de la educación no formal de la infancia y adolescencia, adaptar los criterios educativos constatando que la realidad social se va perfilando con características diferentes ya que la familia ha sufrido numerosos cambios, tanto en su composición como en las relaciones interpersonales. El incremento de la población inmigrante conlleva acciones multiculturales a las que hay que dar salida desde los Ayuntamientos.

Las acciones preventivas y educativas, por su invisibilidad aparente y por la falta de resultados inmediatos, dejan patente el hecho constatable de que en nuestro trabajo la eficacia "se ve por lo que no se ve", nuestro éxito profesional en muchos casos, está en que no haya noticias negativas (e.g., desintegración escolar, problemas de convivencia, conflictos entre iguales, falta de formación en las familias). Es por tanto un trabajo a medio plazo, constante, y solo visible para un observador sensible y comprometido con la calidad de vida de los ciudadanos, que puede plantearse en diferentes posibilidades de intervención educativa en toda la comunidad (con niños, con familias, con profesorado, con personas mayores, prejubilados, jubilados, colectivos y grupos como inmigrantes, población en situación de riesgo psicosocial, absentistas, con necesidades socioeducativas especiales, etc.).

Todo ello hace que esta invisibilidad aparente presenta una cara negativa de cara a baja sensibilidad y reconocimiento social, por un lado, y a de las dificultades de consolidación de estos equipos de su futuro inmediato, por otro. El hecho de que las autoridades locales no se planteasen extraer el máximo provecho educativo de unos equipos que han estado trabajando en algunos casos desde hace 30 años en sus distintas localidades, sería simplemente un despilfarro de los recursos públicos, por lo que esta inversión municipal debería rentabilizarse al máximo. Para ello resulta imprescindible reivindicar estos Servicios como algo específico de los munici- 
pios, y repensar colectivamente la utilidad y funciones que han de tener de cara al conjunto de necesidades de los ciudadanos de los municipios en la España del siglo XXI. Por ello, planteamos las siguientes propuestas:

1. Realizar desde la Coordinadora de Servicios Municipales de Atención Comunitaria Psicológica y Educativa un censo real de los servicios existentes.

2. Confeccionar un banco de datos de los equipos para fomentar su conocimiento, utilizando la Federación Española de Municipios y Provincias como medio de difusión.

3. Establecer un lugar en la red (dentro de la Federación de Municipios y Provincias, a través del Colegio Oficial de Psicólogos, en las páginas de redes relacionadas) un banco de proyectos y experiencias de estos equipos, para que pueda ser utilizado por todos los que lo deseen.

4. Diversas formas de difusión de las experien- cias tanto para los pueblos y ciudades que tengan estos servicios como para los que nos los tengan.

5. Poner los Servicios Psicopedagógicos Municipales al servicio de las políticas locales en materia de educación ciudadana.

6. Propiciar un encuentro estatal para repensar la experiencia de estos servicios, extraer conclusiones válidas para el momento actual y para todos los miembros de la F.E.M.P.

7. Potenciar el papel de los Servicios Psicopedagógicos Municipales mediante su difusión y participación en redes (Red de Ciudades Educadoras, Red a favor de los derechos de la Infancia y la Adolescencia, Coordinadora de Ludotecas Municipales, etc.).

8. Buscar vías para racionalizar y rentabilizar el trabajo y los recursos mediante proyectos técnicos que puedan diseñarse, elaborarse o llevarse a cabo de manera común entre varios municipios.

Manuscrito recibido: $13 / 10 / 2010$

Revisión recibida: 25/11/2010

Manuscrito aceptado: 10/12/2010 